\title{
UV PHOTOABLATION OF ATHEROSCLEROTIC LESIONS. APPLICATION IN CARDIOLOGY
}

\author{
S. AVRILLIER, J. P. OLLIVIER, $\dagger$ I. GANDJBAKHCH, $\dagger$ \\ E. DELETTRE, J. L. BUSSIERE $\dagger$ and E. TINET \\ Laboratoire de Physique des Lasers, Universite Paris XIII 93430, \\ Villetaneuse
}

(Received March 21, 1990; in final form April 5, 1990)

\begin{abstract}
Various methods for the ablation of the atherosclerotic material that fills the coronary arteries are under investigation. Among them laser coronary angioplasty is one of the most promising. In this paper we show that the $\mathrm{XeCl}$ excimer laser $(308 \mathrm{~nm})$ offers many advantages which make it appear as a rather good tool. In vitro studies demonstrate the ability of precise control of the UV photoablation process, there is no thermal damage and the risk of perforation is reduced. A suitable laser-fiber optic delivery system has been developed and the results of the first in vivo procedures are very encouraging.
\end{abstract}

KEY WORDS: Laser angioplasty, excimer laser, photoablation.

\section{INTRODUCTION}

Cardiovascular diseases are the foremost cause of death in France. Narrowing of coronary arteries by atherosclerotic plaque, composed of complex lipid, fibrous and calcified materials, reduces the myocardial vascularization and is often associated with acute infarction.

Although human coronary atherosclerosis is generally a diffuse disease, it lends itself to surgical by-pass procedures because the major site of plaque formation is usually distributed near the aorta. As a result, direct aorta coronary by-pass surgery has become a standardized myocardial revascularization method. On the other hand, in the last decade, percutaneous balloon angioplasty has been a major advance in interventional therapy of coronary obstructions. This technique is less traumatic and less expensive than bypass surgery.

However, the long-term and mid-term results of myocardial revascularization by direct surgical coronary by-pass or percutaneous balloon angioplasty are often compromised by progressive restenosis. Moreover as many as $20 \%$ of coronary arteries are inoperable because of diffuse and/or calcified disease. This problem calls for the development of other techniques: it seems more logical to try to remove the atherosclerotic material that fills the vascular lumen rather than to by-pass it or to flatten it. This idea has led to the finding of various ablative methods using mechanical energy (rotational atherectomy device), ${ }^{1,2}$ ultrasonic energy ${ }^{3}$ or photonic energy with lasers coupled to optical fibers. ${ }^{4}$ Several types of lasers have already been

$\dagger$ H.I.A. du Val de Grâce-74, Bd de Port Royal 75005 Paris. 
tried in in vitro or in vivo studies of atheromatous plaque photoablation and laser angioplasty is already used experimentally as a balloon angioplasty complement or as a self governing method for the recanalization of peripheral and coronary arteries. ${ }^{5}$

The selection of the appropriate laser for coronary angioplasty is based on the following criteria: the quality of the cuts related to the structural integrity of the adjacent tissues and their short term or long term response, the ability of precise control of the ablation process to avoid perforation or dissection, the suitability of the fiber optic delivery system with respect to the fluences required for the arterial plaque ablation, the laser size, reliability and serviceability for current use in hospitals.

\section{UV PHOTOABLATION PROCESS}

Preliminary studies have been achieved with continous wave $(\mathrm{CW})$ lasers like Argon, Nd-YAG or $\mathrm{CO}_{2}$ lasers. ${ }^{6-8}$ Typically thermal diffusion during $\mathrm{CW}$ ablation process leads to necrosis, coagulation and gross charring of the surrounding tissues. ${ }^{7,9-11}$ The use of a pulsed mode at low repetition rate and at high energy widely reduces these drawbacks. ${ }^{12}$ Another important characteristic is the laser wavelength which determines the penetration depth of the radiation: a small etch depth per laser pulse provides a better control of the ablation process.

Therefore, the excimer lasers, operating in the UV region and strongly absorbed by arterial tissues, are particularly adapted to angioplasty ${ }^{13,14}$ since they deliver their energy in few tens nanosecond pulses. Early studies about the interaction between UV light and polymers have demonstrated that UV photonic energy can induce localised electronic excitation and molecular bindings disruption leading to the so-called "UV photoablation phenomenon." 15 UV photoablation of polymers or biological materials has been observed for a number of excimer lasers wavelengths such as $193 \mathrm{~nm}(\mathrm{ArF}), 248 \mathrm{~nm}(\mathrm{KrF}), 308 \mathrm{~nm}(\mathrm{XeCl})$ and $351 \mathrm{~nm}(\mathrm{XeF}) \cdot{ }^{15-18}$ Above a threshold fluence, the cuts are very well defined: the section is nearly equal to the incident beam section and the cuts edges are very sharp. The etch depth per pulse, depending on the material, is of the order of a few tens micron (Figure 1).

The mechanism of UV photoablation is not yet completely understood. ${ }^{19}$ After the absorption of a UV photon, generating electronic excitation, many relaxation channels are possible like direct decomposition in primary products or internal relaxation leading to heat dissipation or bond breaking.

The kinetic of this phenomenon is obviously very complicated, but, as a general trend, one can say that thermal relaxation prevails at low irradiation intensity whereas an efficient degradation with a small but proportional production of heat is obtained at high irradiation intensity.

UV photoablation depends on the radiation wavelength and the power density but also on the nature of the irradiated material. For biological tissues, the differences between the samples and the pathological state diversity lead to a large variability in the measured cutting rates. In fact, it is very difficult to predict precisely the ablation efficiency for a new sample. 


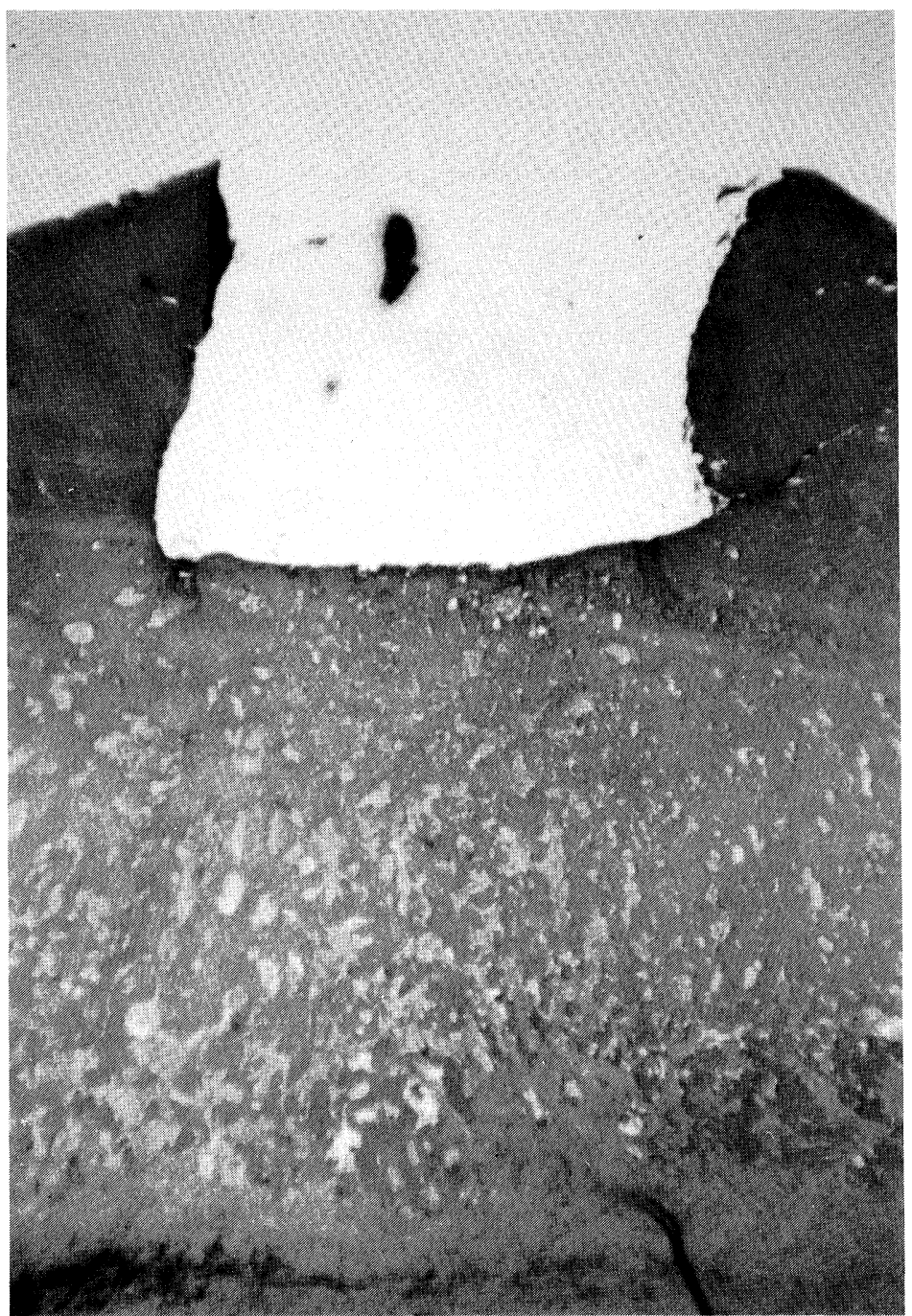

Figure 1 Microphotograph of post-mortem human atherosclerotic aorta: effect of $20 \mathrm{XeCl}$ laser pulses of $12 \mathrm{~mJ}$ each and $20 \mathrm{~ns}$ duration of $\mathrm{XeCl}$ radiation delivered through a $600 \mu \mathrm{m}$ core fiber in contact with the arterial wall. The fiber was directed perpendicular to the internal surface of the artery. The incision depth is about $450 \mu \mathrm{m}$ and the width about $550 \mu \mathrm{m}$. There is no thermal injury on the incision margins and the cut edges are very sharp.

This process obviously cannot be compared to pure thermal vaporization: for example, in air and with a $20 \mathrm{~ns}$ pulse of $12 \mathrm{~mJ}$ at $308 \mathrm{~nm}$ the ablated volume in the arterial wall is usually of the order of $1.5 \times 10^{-2} \mathrm{~mm}^{3,20}$ which corresponds to a specific ablation energy of $0.8 \mathrm{~J} / \mathrm{mm} .{ }^{3}$ It is interesting to notice that the specific energy of tissue heating from room temperature to $100^{\circ} \mathrm{C}$ plus its vaporization (assuming that the tissue's thermal properties are the same as those of water) is $2.8 \mathrm{~J} / \mathrm{mm}^{3}$ that is about 3.5 times the energy required for $308 \mathrm{~nm}$ photoablation. 
The quality of the incisions probably arises from the fact that the radiation is absorbed on a short depth and that any excess energy over that required to break the bounds remains in the ejected products and is dissipated in the surrounding medium.

In an extensive in vitro quantitative study of $308 \mathrm{~nm}$ post-mortem human aortic walls photoablation ${ }^{20,21}$ we have measured the cutting rates in different media (air, saline and blood) as a function of the incident energy density and estimated the threshold fluences.

Internal surface of fresh postmortem human atherosclerotic aortic fragments were irradiated by the mean of a $600 \mu \mathrm{m}$ core diameter fiber coupled to a $\mathrm{XeCl}$ laser. The energy density per pulse at the output of the fiber was varied from 1.4 to $7 \mathrm{~J} / \mathrm{cm}^{2}$ and the segments, irradiated by a series of $5-100,20 \mathrm{~ns}$ pulses, were submitted to histologic examination.

In spite of the large variability in measured properties due to differences between the pathological states of the samples, we could establish several important points out of the results of this study:

$-308 \mathrm{~nm}$ photoablation of the plaque, even calcified, can be obtained efficiently in air but also in saline or in blood. For example, with 20 pulses of $12 \mathrm{~mJ}$ delivered by a $600 \mu \mathrm{m}$ core diameter fiber in contact with the sample, the cut depths in the fibrous intimal and medial layers of an aortic human artery have been found to be respectively $990 \mu \mathrm{m}, 850 \mu \mathrm{m}$ and $810 \mu \mathrm{m}$ in air, saline and blood.

- The ablation threshold fluences for $20 \mathrm{~ns}$ pulses have been evaluated at $1.4 \mathrm{~J} / \mathrm{cm}^{2}$ in air and in blood. However, in saline the threshold seems to be slightly higher and close to $1.7 \mathrm{~J} / \mathrm{cm}^{2}$.

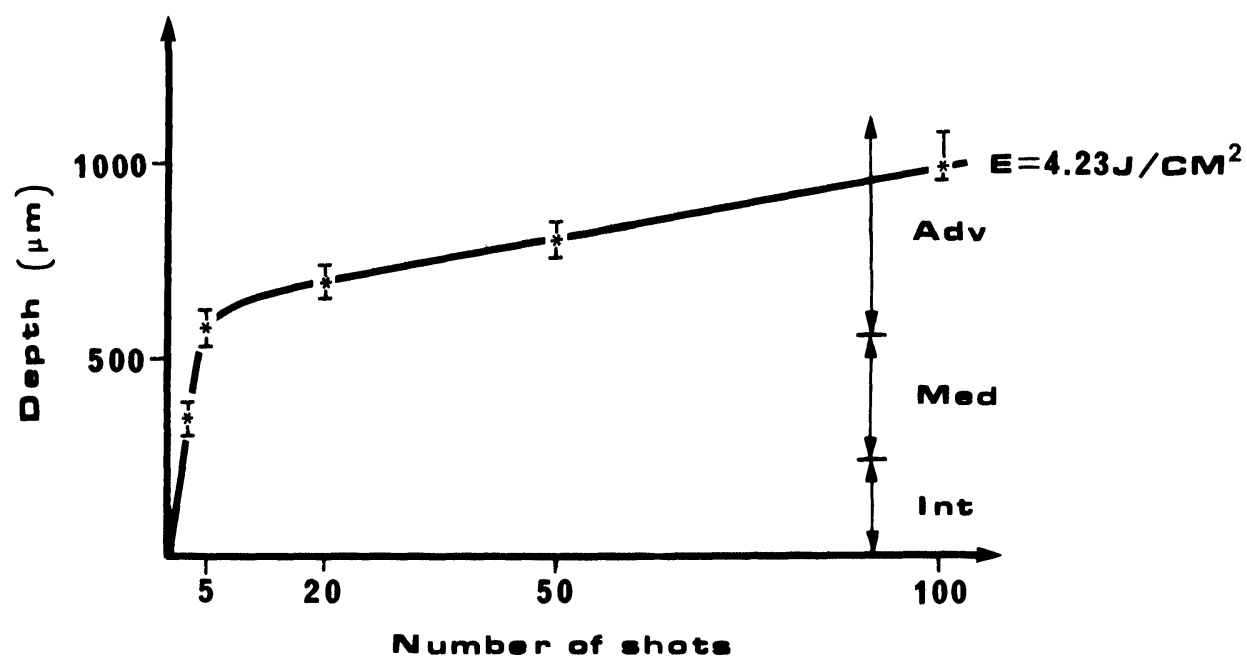

Figure $2 \mathrm{XeCl}$ laser ablation depth as a function of the number of shots for a constant density energy of $4.23 \mathrm{~J} / \mathrm{cm}^{2}$. The sample is a highly fibrous human aortic fragment. The thickness of each arterial layer is indicated (intima, media, adventice). The cutting rate is much smaller in the adventice than in the intima or in the media: the adventical tissue is reached after only five pulses and a perforation is obtained over 100 pulses. This type of curve is also observed for all arteries in saline and blood. 
-The ablation efficiency increases with the energy density and the thermal damaged layer on the cut edges never exceeds $50 \mu \mathrm{m}$.

-In liquid media as saline or blood small disruptions of the intimal layer may appear and are probably due to a shock wave induced in the medium by the laser pulse.

-In all media we have demonstrated a clear resistance of the artery wall adventicial (external) layer to $308 \mathrm{~nm}$ photoablation. This phenomenon, probably due to a much higher threshold fluence of this tissue, is a very important safety factor for the use of $\mathrm{XeCl}$ laser in human coronary angioplasty (Figure 2).

\section{LASER DELIVERY SYSTEM AND FIRST IN VIVO PROCEDURES}

Many $\mathrm{XeCl}$ lasers are commercially available. Their operation is easy and reliable and in opposition to other excimer lasers ( $\mathrm{KrF} 249 \mathrm{~nm}, \operatorname{ArF} 193 \mathrm{~nm}, \mathrm{XeF} 351 \mathrm{~nm})$ no gas filtration is needed. However their usual size and running cost may still be a problem for clinical use.

For our in vitro and in vivo experiments we employ now a new generation of excimer lasers (SOPRA) using a photon switching technique. The laser head is very compact $(994 \mathrm{~cm} \times 34 \mathrm{~cm} \times 25 \mathrm{~cm})$ and delivers, in its original design, $300 \mathrm{~mJ}$ pulses of duration $40 \mathrm{~ns}$ at $308 \mathrm{~nm}$. Modifying the discharge impedance we increased the pulse duration up to $100 \mathrm{~ns}$ FWHM with an output pulse energy of $120 \mathrm{~mJ}$ at a repetition rate of $3 \mathrm{~Hz}$. In a preliminary systematic study we found out that the coupling efficiency of $308 \mathrm{~nm}$ laser beam into optical fibers is much more dependent on the beam quality than on the pulse duration.

Table 1 summarises the values of the maximum energy per pulse that can be reliably coupled into some commercial fibers (HCN Ensign Bickford) with our new apparatus: the laser beam $(20 \mathrm{~mm} \times 20 \mathrm{~mm}$ cross section $)$ is simply focused by a $100 \mathrm{~mm}$ lens on the cleaved and striped tip of carefully aligned $1 \mathrm{~m}$ long fiber. It can be seen that, for a $1 \mathrm{~mm}$ core diameter, $75 \%$ of the laser power is available at the output of the fiber. The fluences at the distal tip are 8-12 times higher than the plaque ablation threshold $\left(1.4 \mathrm{~J} / \mathrm{cm}^{2}\right)$. For angioplasty the optimum operating fluence is of the order of 6 or $7 \mathrm{~J} / \mathrm{cm}^{2}$ to prevent damage to the distal end of the fiber and to minimize thermal and acoustic damage to the arterial wall.

For clinical applications we integrated the laser head, power supply, vacuum pump, gas mixture bottles and the fiber optical coupling device to a movable self contained medical apparatus $(120 \mathrm{~cm} \times 60 \mathrm{~cm} \times 100 \mathrm{~cm})$.

Table 1 Experimental values of $\mathrm{XeCl}$ laser energy and fluences coupled into various core diameter $\mathrm{HCN}$ fibers. $100 \mathrm{~ns}$ duration laser pulses. $1 \mathrm{~m}$ long fibers

\begin{tabular}{lll}
\hline $\begin{array}{l}\text { Core diameter } \\
(\mu \mathrm{m})\end{array}$ & $\begin{array}{l}\text { Output energy per pulse } \\
(\mathrm{mJ})\end{array}$ & $\begin{array}{l}\text { Energy density per pulse } \\
\left(\mathrm{J} / \mathrm{cm}^{2}\right)\end{array}$ \\
\hline 1,000 & 90 & 11.5 \\
600 & 48 & 17 \\
400 & 19 & 15.1 \\
200 & 4 & 12.7 \\
\hline
\end{tabular}




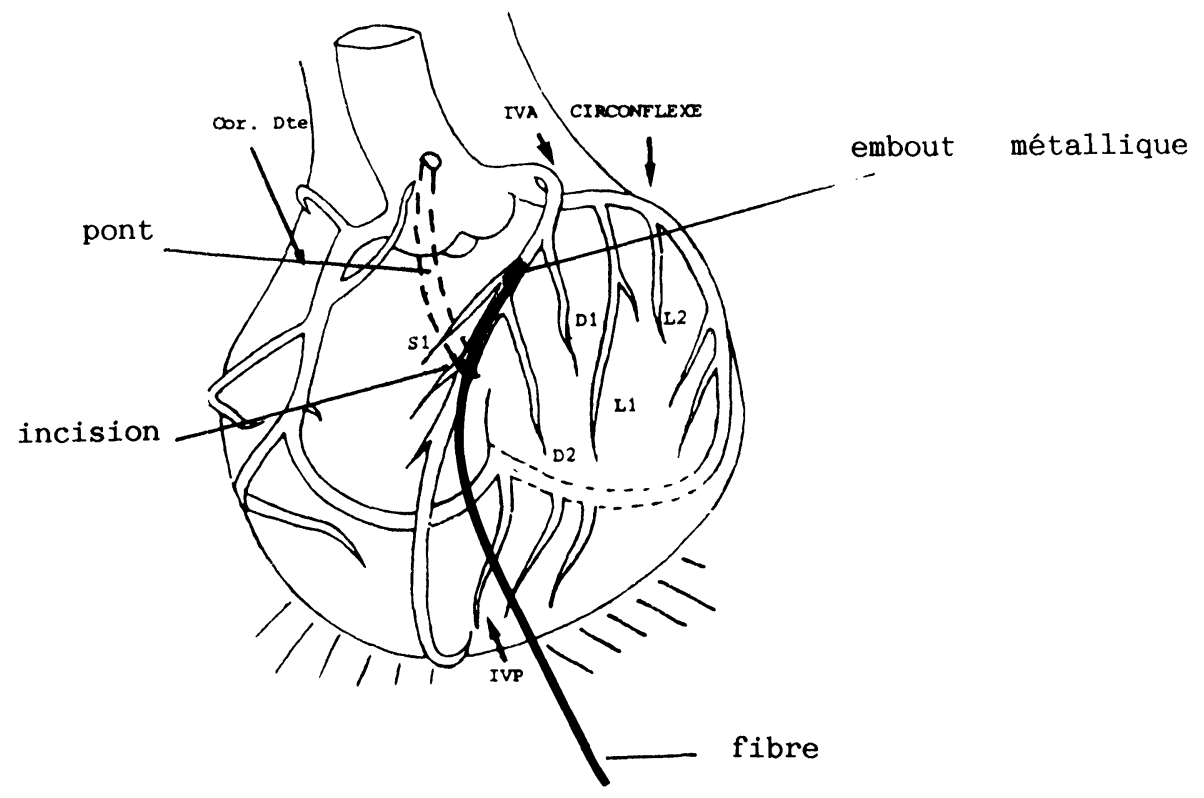

Figure 3 Prior to by-pass grafting, a $1 \mathrm{~mm}$ core diameter specifically tipped fiber was introduced via the coronary arteriotomy and directed in contact with atheromatous lesion upstream or downstream from the by-pass graft implantation site.

The necessities of performing the first $\mathrm{XeCl}$ laser angioplasties with utmost safety and to undertake complementary endarterectomy in many patients, led us, since June 1988, to achieve a number of angioplasties during coronary artery by-pass surgery. ${ }^{22,23}$ Prior to by-pass grafting, a $1 \mathrm{~mm}$ core diameter specifically tipped fiber was introduced via the coronary arteriotomy and directed in contact with atheromatous lesion upstream or downstream from the by-pass graft implantation site (Figure 3). A successful 1-20 mm long endarterectomy was achieved in 11/12 segments through 100-3200, 16-28 mJ pulses. In both 8th day and 6th month angiocoronarographic controls, all laser treated segments showed a good patency, except in one. No immediate complications were observed except a small intimal haemorrhage, observed in patient 2 , that could have been secondary to the fracture of the plaque remaining at the periphery of the vessel lumen.

Even if the number of procedures performed in this preliminary study is too small to predict the long term effects of the laser procedure, one can now state that intraoperative $\mathrm{XeCl}$ laser endarterectomy is no more a trial field for the percutaneous method, but a proper therapeutic method. In addition to by-pass surgery, this method can be used to perform downstream complementary angioplasty, desobstruction of not graftable highly calcified coronary arteries and microangioplasty of small diameter coronary arteries. Collateral secondary arteries can also be re-irrigated even in the by-passed segment: moreover the specific indications of by-pass surgery could be extended to the treatment of very calcified lesions and the reopening of total distal occlusions. 


\section{FUTURE DEVELOPMENTS OF THE TECHNIQUE}

After the first series of 12 endarterectomies, the need of various catheters with different shapes, diameters and flexibilities became apparent. This to allow the surgeon to choose the best matching catheter characteristics relative to the artery being treated. For this reason, we decided to deliver the laser power at the operation site with a $1 \mathrm{~mm}$ core diameter fiber and to connect, at the distal tip of this fiber, any short and convenient fiber optic catheter (see Figure 4). The $100 \mathrm{~mJ}$ which are available at the output of the main $1 \mathrm{~mm}$ core diameter fiber can be coupled to several models of catheters shown in Figure 5. For example, one of the catheters developed in our laboratory uses $17200 \mu \mathrm{m}$ fibers and has an external diameter of 1,6 mm. In this particular catheter we coupled a total energy of $30 \mathrm{~mJ}$ per pulse, which corresponds to an average fluence of $5,6 \mathrm{~J} / \mathrm{cm}^{2}$ at the output core of each $200 \mu \mathrm{m}$ fiber.These new catheters will be tested in the near future during by-pass surgery.

Concerning percutaneous $\mathrm{XeCl}$ coronary angioplasty and in order to be able to follow a percutaneous route, we have developed a family of multifiber flexible catheters: The small diameter fibers $(100-300 \mu \mathrm{m})$ are concentrically arranged around a lumen for a guide wire: by first advancing the guide through tortuous vascular segments it is then possible to position the catheter tip in contact with the lesion. For example, one of the catheters developed in our laboratory uses 16 individual $200 \mu \mathrm{m}$ fibers and has an external diameter of $2 \mathrm{~mm}$. In June 1989 Litvack et al. ${ }^{24}$ had already treated 63 patients with $76 \%$ primary success. Most of the

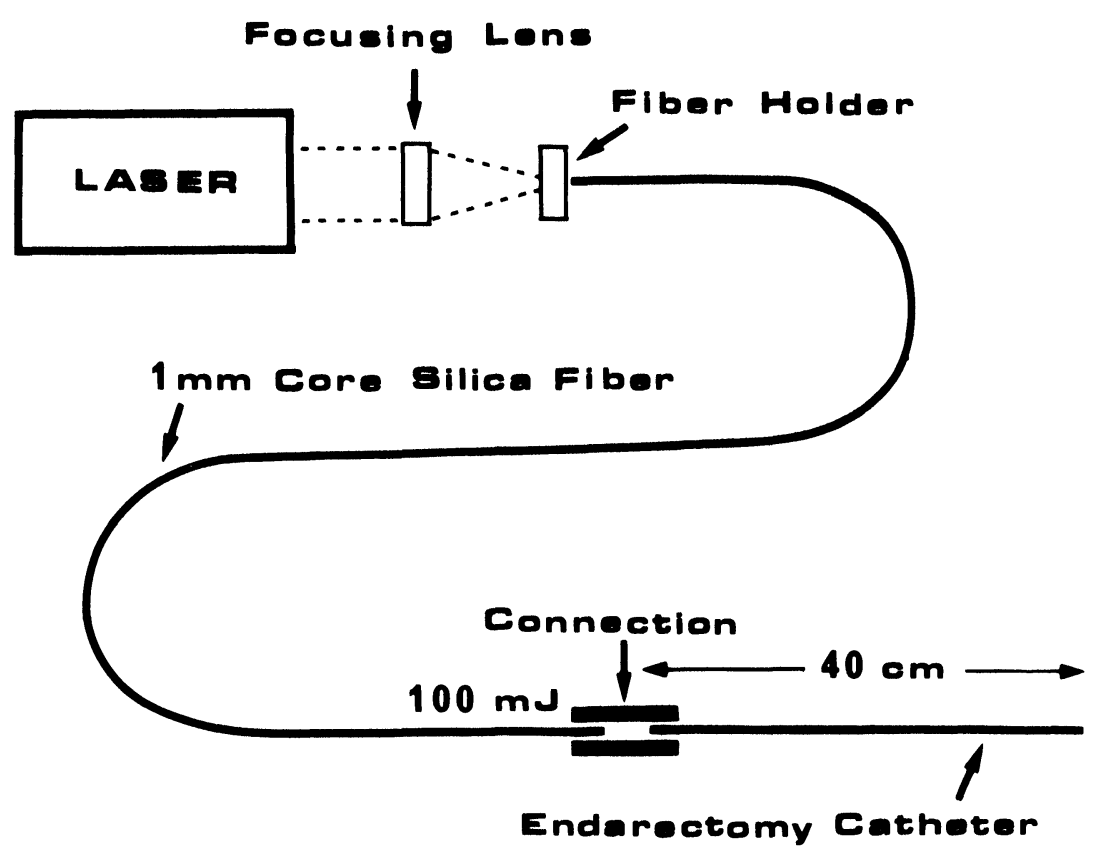

Figure 4 The laser power is delivered at the operation site by a $1 \mathrm{~mm}$ core diameter fiber and any short and convenient fiber catheter is connected at the distal tip of this fiber. 


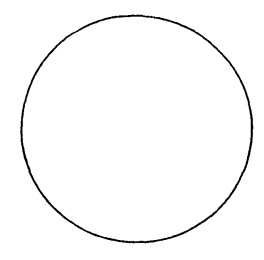

\section{Bingle Optical Fiber Catheters \\ $0.4<\Phi<1.5 \mathrm{~mm}$}

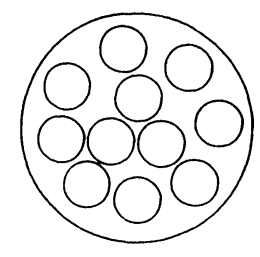

Full Filled Multifiber Catheters

$0.7<\Phi<2 \mathrm{~mm}$

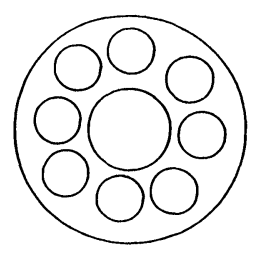

\section{Multifiber Catheters with \\ Contral Lumen \\ $1.7<\Phi<3 \mathrm{~mm}$}

Figure 5 Various catheters developed in our laboratory.

immediate failures seem to be due to a lack of flexibility of the catheters and the complications are mainly vascular dissection (mechanical or photoablative?) and vascular spasms. The middle or long term healing process remains unknown.

A lot of work remains to be done to improve intra-operative and percutaneous coronary artery angioplasty. A better optical quality of the laser beam could lead to easier and more reliable energy coupling into the fibers. The repetition rate should be increased to 20 or $50 \mathrm{~Hz}$ with the same output energy per pulse reducing the duration of the operations. The multifiber catheters must be modified to improve their flexibility and their adequacy to clinical procedures. A more accurate over the in situ ablation process would also increase the safety of this method. In intraoperative procedures this could be achieved by direct angioscopy visualization using one channel of the angioplasty catheter because there is no circulating blood.

In percutaneous procedures, a technique of spectral identification of the atherosclerotic tissue by the analysis of the fluorescence induced by the laser beam is under investigation in our laboratory. Yet, up to the present, the results of this study do not indicate that such a diagnostic would be safe enough for a current use in hospitals. Finally, the usefulness of $\mathrm{XeCl}$ angioplasty will be stated only if larger series of cases demonstrate a real improvement of long term results, for which the rate of restenosis still appears as the main point. 


\section{CONCLUSION}

Since the first reports on the in vitro effect of $\mathrm{XeCl}$ excimer laser energy on human vascular tissue in $1985,{ }^{25,26}$ the story of human coronary $308 \mathrm{~nm}$ angioplasty, sustained by many thorough investigations, has been very fast and marked by an amazing convergence of favourable factors: $\mathrm{XeCl}$ excimer laser photoablation process supports controlled submillimeter removal of atherosclerosis plaques, even calcified, with no or minimal damage to neighbouring tissues. The risk of perforation is reduced by a clear resistance of the adventitial layer. There is no evidence for $308 \mathrm{~nm}$ induced carcinogenesis. $308 \mathrm{~nm}$ radiation does not induce blood coagulation and is able to liquefy clots. $\mathrm{XeCl}$ lasers of reasonable size, cost and serviceability can be coupled to flexible multifiber catheters in order to safely deliver the required fluences. And finally, the first in vivo human investigations are very promising.

\section{References}

1. K. Kensey, J. Nash, C. Abrahams, K. Lake and C. K. Zarins. Recanalisation of obstructed arteries using a flexible rotating tip catheter (abstract), Circulation (Suppl II), 74, 457 (1986).

2. N. Zacca, A. Raizner, D. Short, G. Noon, C. D. Weilbaecherd, J. Roehm, A. Gotto and R. Roberts. First in vivo human experience with a recently developed rotational atherectomy device (abstract), Circulation (Suppl IV), 76, 46 (1987).

3. R. J. Siegel, M. C. Fishbein, J. Forrester, K. Moore, E. de Castro, L. Daykhovsky and T. A. Don Michael. Ultrasonic plaque ablation, a new method for recanalisation of partially or totally occluded arteries, Circulation, 78, 1448-1451 (1988).

4. J. S. Forrester, Laser angioplasty: now and the future, Circulation, 78, 777-779 (1988).

5. For an extensive bibliography, see for example 4 and T. A. Sanborn. Laser angioplasty. What has been learned from experimental studies and clinical trials? Circulation, 78, 769-774 (1988).

6. D. S. J. Choy, S. H. Stertzer, H. Z. Rotterdam and M. S. Bruno. Laser coronary angioplasty : experience with 9 cadaver hearts. Am. J. Cardiol. 50, 1209-1211 (1982).

7. G. S. Abela, S. Normann, D. Cohen, R. L. Feldman, E. A. Geiser and R. Conti, Effects of carbon dioxide, $\mathrm{Nd}$ Yag and argon laser radiation on coronary atheromatous plaques. Am. J. Cardiol. 50, 1199-1205 (1982).

8. J. P. Ollivier, P. Rossant, I. Gandjbakhch, B. Warme-Janville, A. Quenzer, R. Brion et Droniou. Effets de differents types de lasers a emission continue sur les plaques athéromateuse humaines in vitro. Arch. Mal. Coeur. 4, 554-558 (1985).

9. J. F. Hiehle, D. B. C. Bourgelais, S. Shapshay, F. J. Schoen, D. Kim and R. Spears. Nd-Yag laser fusion of human atheromatous plaque. Arterial wall separations in vitro. Am. J. Cardiol. 56, 953-957 (1985).

10. G. Lee, R. Ikeda, I. Herman, R. M. Dwyer, M. Bass, H. Hussein, J. Kozina and D. T. Mason. The qualitative effects of laser irradiation on human arteriosclerotic disease. Am. Heart. J. 105, 885-889 (1983).

11. J. M. Isner, R. F. Donaldson, J. T. Funai, L. I. Deckelbaum, N. G. Pandian, R. H. Clarke, M. A. Konstam, D. M. Salem and J. S. Bernstein. Factors contributing to perforations resulting from laser coronary angioplasty: observations in an intact human postmortem preparation of intraoperative laser coronary angioplasty. Circulation, 72, 191-199 (1985).

12. L. I. Deckelbaum, J. M. Isner, R. F. Donaldson, R. H. Clarke, S. Laliberte, A. S. Aharon and J. S. Bernstein. Reduction of laser-induced pathologic injury using pulsed energy delivery. Am. J. Cardiol. 56, 662-667 (1985).

13. R. Linsker, R. Srinivasan, J. J. Wynne and D. R. Alonso. Far-ultraviolet laser ablation of atherosclerotic lesions. Lasers. Surg. Med. 4, 201-206 (1984).

14. D. L. Singleton, G. Paraskevopoulos, R. S. Taylor and L. A. J. Higginson. Excimer laser angioplasty: tissue ablation, arterial response and fiberoptic delivery. IEEE J. Quantum Electron, QE23, 1772-1782 (1987). 
15. R. Srinivasan and W. J. Leigh. Ablative photodecomposition: action of far-ultraviolet (193 nm) laser radiation on poly(ethylene terephthalate) films. J. Am. Chem. Soc. 104, 6784-6785 (1982).

16. G. Koren and J. T. C. Yeh. Emission spectra surface quality and mechanism of excimer laser etching of polyimide films. Appl. Phys. Lett. 44, 1112-1114 (1984).

17. G. Koren, J. T. C. Yeh. Emission spectra and etching of polymers and graphite irradiated by excimer lasers. J. Appl. Phys. 56, 2120-2126 (1984).

18. P. E. Dyer and J. Sidhu. Excimer laser ablation and thermal coupling efficiency to polymer films. $J$. Appl. Phys. 57, 1420-1422 (1985).

19. S. Lazare and V. Granier. Ultraviolet laser photoablation of polymers: a review and recent results. Laser Chem. 10, 25-40 (1989).

20. S. Avrillier, J. P. Ollivier, E. Raynal, J. P. Berthier and Y. Rougier. Excimer laser angioplasty: quantitative study. International conference on photodynamic therapy and medical laser applications. London July 1988.

21. E. Delettre, S. Avrillier, J. P. Ollivier, J. P. Berthier, E. Raynal and Y. Rougier. In vitro quantitative study of $\mathrm{XeCl}$ laser angioplasty: medium effects and adventrical resistance to photoablation, submitted to Lasers in Med. Science.

22. J. P. Ollivier, S. Avrillier, I. Gandjbakhch, E. Raynal, J. Debourayne and J. Droniou. In vivo excimer laser coronary angioplasty in humans: preliminary results. Eur. Heart J. 9, 331 (1988).

23. J. P. Ollivier, I. Gandjbakhch, S. Avrillier, E. Delettre, J. L. Bussiere and C. Cabrol. Intraoperative excimer laser coronary artery endarterectomy. Thorac. Cardio. Vasc. Surg. (In press).

24. F. Litvack, W. Grundfest and J. C. Forrester. Percutaneous excimer laser coronary angioplasty (ELCA). Preliminary experience, "Prospective en chirurgie vasculaire". Marseille Sept. 1989.

25. W. S. Grundfest, F. Litvack, J. S. Forrester, T. Goldenberg, H. J. C. Swan, L. Morgenstein, M. Fishbein, S. McDermid, D. M. Rider, T. J. Pacala, J. B. Laudenslager. Laser ablation of human atherosclerotic plaque without adjacent tissue injury. JACC, 5, 929-933 (1985).

26. G. A. Abil'siitov, A. A. Belyaev, M. A. Bragin, E. P. Velikhov, V. S. Zhdanov, T. I. Karu, V. S. Letokhov, S. E. Ragimov, M. Y. Ruda, A. V. Trubetskoi, N. P. Furzikov and E. I. Chazov. Investigation of photoablation of atherosclerotic plaques by laser radiation. Sov. J. Quantum Electron, 15, 1314-1316 (1985). 